\title{
Vai trò trung gian của sự tương thích giá trị trong mối quan hệ giữa tính cách chủ động và hành vi giúp đõ̃ của nhân viên
}

\section{The mediation role of value congruence in the relationship between proactive personality and helping behavior}

\author{
Nguyễn Thế Khải ${ }^{1 *}$, Trịnh Thuỳ Anh ${ }^{1}$, Đỗ Thị Thanh Trúc ${ }^{1}$, Nguyễn Hoàng Nguyên ${ }^{2}$ \\ ${ }^{1}$ Trường Đại học Mở Thành phố Hồ Chí Minh, Việt Nam \\ ${ }^{2}$ Trường Đại học Yersin Đà Lạt, Việt Nam \\ *Tác giả liên hệ, Email: khai.nt@ou.edu.vn
}

THÔNG TIN

DOI: $10.46223 / \mathrm{HCMCOUJS}$. econ.vi.16.1.644.2021

Ngày nhận: 12/08/2020

Ngày nhận lại: 18/08/2020

Duyệt đăng: 06/01/2021

\section{Tù khóa:}

hành vi giúp đỡ, sự tương thích giá trị với tồ chức, sự tương thích giá trị với người quản lý trực tiếp, tính cách chủ động

Keywords:

helping behavior, personorganization value congruence, personsupervisor value congruence, proactive personality

\section{TÓM TẮT}

Nghiên cứu được thực hiện nhằm tìm hiểu tác động của tính cách chủ động (proactive personality - PP) đối với hành vi giúp đỡ của nhân viên (helping behavior -HB) thông qua hai biến trung gian là sự tương thích giá trị với tổ chức (Person-Organization Value congruence - POV), và sự tương thích giá trị với người quản lý trực tiếp (Person-Supervisor Value congruence- PSV). Với dữ liệu được thu từ 715 nhân viên điều dưỡng tại các bệnh viện tại Việt Nam, nghiên cứu phân tích nhân tố khẳng định (CFA), hồi quy đa biến bằng Process macro. Kết quả cho thấy $\mathrm{PP}$ có tác động tích cực với POV, PSV và HB. Ngoài ra, kết quả phân tích bootstrap cho thấy $\mathrm{POV}, \mathrm{PSV}$ có vai trò trung gian trong mối quan hệ giữa $\mathrm{PP}$ và $\mathrm{HB}$. Nghiên cứu nhấn mạnh tầm quan trọng của $\mathrm{PP}$ trong việc phát triển sự tương thích giá trị với tổ chức và người giám sát trực tiếp cũng như hành vi giúp đỡ của nhân viên. Lý thuyết, ý nghĩa thực tiễn và hạn chế của nghiên cứu cũng được thảo luận nhằm giúp các nhà nghiên cứu và nhà quản trị hiểu rõ hơn về mối quan hệ giữa các yếu tố trong mô hình nghiên cứu và có các biện pháp quản trị phù hợp.

\section{ABSTRACT}

The study was conducted to investigate the impact of proactive personality (PP) on the helping behavior (HB) through the mediation of Person-Organization Value congruence (POV), and PersonSupervisor Value congruence (PSV). With data collected from 715 nursing staffs at hospitals in Vietnam, the research affirmative factor analysis (CFA), multivariate regression by Process macro. The results showed that PP has a positive effect on POV, PSV and HB. In addition, the results of the bootstrap analysis showed that POV, PSV serially mediate the effect of PP on HB. The study underscores the importance of PP in developing a value compatible with the organization and its direct supervisors as well as actively helping employees. The theory, practical implications and limitations of the research are also discussed. 


\section{Giới thiệu}

Trong môi trường kinh doanh đầy biến động và cạnh tranh ngày càng gay gắt hiện nay, các tổ chức mong đợi nhân viên thể hiện hành vi chủ động để đạt được thành công cả về cá nhân và tổ chức (McCormick, Guay, Colbert, \& Stewart, 2019). Do đó, bên cạnh việc nhấn mạnh giá trị tổ chức của mình đến nhân viên, các tổ chức cần coi hành vi là tự khởi xướng, định hướng thay đổi và định hướng tương lai để tạo sự thay đổi tích cực trong tổ chức là một phần của công việc, và tổ chức cũng cần nỗ lực tìm kiếm các nhân viên có định hướng chủ động (Bindl \& Parker, 2011). Bởi những người có tính cách chủ động là những người kiểm soát tình huống và tích cực thúc đẩy sự thay đổi trong môi trường của họ (Bateman \& Crant, 1993). Nắm bắt xu hướng này, các nhà nghiên cứu đã tìm cách làm sáng tỏ cấu trúc của hành vi chủ động ở cả cấp độ cá nhân và cấp độ nhóm (Bateman \& Crant, 1993; Bindl \& Parker, 2011; Grant \& Ashford, 2008; Griffin, Neal, \& Parker, 2007).

Hành vi chủ động đã thu hút sự quan tâm của các nhà nghiên cứu và báo chí (DuBrin, 2012) nhưng nó vẫn có những khi vực tiềm năng để nghiên cứu thêm. Đầu tiên, Parker, Bindl, và Strauss (2010) tóm tắt là hành vi chủ động gồm năm loại: (1) đảm nhiệm (taking charge), (2) giúp đỡ (helping), (3) lên tiếng (voice), (4) đổi mới cá nhân (individual innovation), và (5) ngăn ngừa sai sót (problem prevention). Các hành vi chủ động như đảm nhiệm, giúp đỡ, lên tiếng và đổi mới cá nhân đã xuất hiện trong rất nhiều nghiên cứu (Xu, Qin, Dust, \& DiRenzo, 2019), tuy nhiên các nghiên cứu về hành vi giúp đỡ mới chỉ giới thiệu khái niệm và đặc điểm phân loại hành vi này, các nghiên cứu thực nghiệm về hành vi giúp đỡ vẫn còn hạn chế. Điều này cho thấy nhiều nghiên cứu đã chứng minh vai trò quan trọng của $\mathrm{PB}$, nhưng hiểu biết về $\mathrm{HB}$ vẫn còn chưa thống nhất.

Nghiên cứu tổng hợp về tính cách chủ động cho thấy yếu tố này tác động đến nhiều kết quả tích cực như sự hài lòng công việc, gắn kết với tổ chức, thành công nghề nghiệp và hiệu quả công việc (Allen, 2012; Christian, Garza, \& Slaughter, 2011; Ng \& Feldman, 2012; Ng \& Feldman, 2014; Rauch \& Frese, 2007). Tuy nhiên, lý thuyết tồn tại (extant theory) (Lewin \& Cartwright, 1964) lại cho rằng đặc điểm cá nhân lại không ảnh hưởng đến hành vi nhiều bằng đặc điểm cá nhân tương tác với môi trường sẽ tác động mạnh đến việc thể hiện hành vi của cá nhân. Vì vậy, các tổ chức không chỉ đơn thuần chọn những nhân viên có tính cách chủ động và mong chờ họ thể hiện hành vi chủ động mà cần quan tâm đến các yếu tố môi trường tổ chức để thúc đẩy nhân viên thể hiện các hành vi chủ động.

Hơn nữa, để đáp ứng được sự thay đổi liên tục của môi trường bệnh viện các nhà quản lý thường quan tâm nhiều hơn đến sự linh hoạt của nhân viên điều dưỡng trong việc thích ứng với những thay đổi của hoàn cảnh và cam kết của họ đối với tổ chức. Nghiên cứu của Kristof-Brown, Zimmerman, và Johnson (2005) và Chen, Yen, và Tsai (2014) chỉ ra rằng sự tương thích giá trị với tổ chức và người quản lý trực tiếp có ảnh hưởng tích cực đến hành vi và hiệu quả công việc. Tuy nhiên, có ít nghiên cứu nào nghiên cứu mối quan hệ giữa tính cách chủ động và sự tương thích giá trị cũng như tác động của sự tương thích giá trị trong quá trình mà tính cách chủ động dẫn đến hành vi giúp đỡ.

Nghiên cứu này đề xuất sự tương đồng giá trị đóng vai trò trung gian trong mối quan hệ giữa tính cách chủ động và hành vi giúp đỡ. Dựa vào mô hình Job Demand-Resource (Bakker \& Demerouti, 2007) nghiên cứu thiết lập mô hình đa biến trung gian (multiple mediation model) trong đó $\mathrm{POV}$ và $\mathrm{PSV}$ đóng vai trò trung gian trong mối quan hệ giữa $\mathrm{PP}$ và $\mathrm{HB}$. Mô hình này cung cấp sự hiểu biết sâu sắc hơn về cơ chế tương tác giữa tính cách và môi trường dẫn đến hành vi giúp đỡ của tính cách chủ động. 


\section{Cơ sở lý thuyết và giả thuyết nghiên cứu}

\subsection{Lý thuyết nền}

Tett và Burnett (2003) đã phát triển thuyết xu hướng hành động (Trait Activation Theory - TAT) để giải thích các yếu tố tình huống có thể tác động đến xu hướng hành động của những tính cách khác nhau. Tett và Burnett nhấn mạnh hai điểm chính liên quan đến việc sử dụng tính cách trong việc dự đoán kết quả làm việc: 1) đặc điểm là thiên hướng hoặc tiềm năng tiềm ẩn vốn có của một cá nhân được kích hoạt bởi môi trường và 2) để đánh giá đúng các hành vi cần quan tâm đến các đặc điểm tình huống có liên quan. Thuyết xu hướng hành động cung cấp nền tảng cho mục tiêu nghiên cứu bằng việc xác định rằng sự khác biệt cá nhân dựa trên tính cách tác động đến hành vi chủ động thông qua những tác động tình huống.

Theo Bardi và Schwartz (2003) các giá trị cá nhân không chỉ định hướng hành vi mà còn là yếu tố tác động đến các hành vi. Giá trị của con người hình thành nên một hệ thống thứ tự các ưu tiên theo từng đặc điểm cá nhân. Trật tự theo thứ bậc của giá trị cũng góp phần phân biệt giá trị với phong tục hay thái độ. Tầm quan trọng tương đối của nhiều giá trị khác nhau định hướng hành động. Mỗi thái đổi hay hành vi về cơ bản có ảnh hưởng đến nhiều hơn một giá trị. Trong số những sự đánh đổi này, những giá trị mâu thuẫn sẽ định hướng thái độ và hành vi (Schwartz, 1996, 1992). Giá trị ảnh hưởng đến hành động khi chúng có mối liên quan trong bối cảnh và quan trọng với hành vi của cá nhân (Schwartz et al., 2012).

Bên cạnh đó, nghiên cứu sử dụng thuyết hành vi được hoạch định (Theory of Planned Behaviour - TPB) (Ajzen, 1991) để giải thích mối liên hệ giữa giá trị và hành vi. TPB giả định thái độ và chuẩn chủ quan của cá nhân và nhận thức kiểm soát hành vi tác động đến ý định thực hiện hành vi.

\subsection{Mối quan hệ giữa tính cách chủ động và sụ tương thích giá trị với tổ chức}

Trong thập kỷ gần đây các nhà nghiên cứu dành sự quan tâm và có nhiều nghiên cứu về các các giá trị của cá nhân và tổ chức lấy cảm hứng từ tính cách chủ động của nhân viên (Vveinhardt \& Gulbovaite, 2014). Các nghiên cứu thực nghiệm của Vveinhardt và Gulbovaite (Vveinhardt \& Gulbovaite, 2013, 2014) đã cho thấy tầm quan trọng của sự tương thích giá trị giữa nhân viên và tổ chức. Kết quả nghiên cứu cho thấy sự tương thích giá trị giữa nhân viên và tổ chức có liên kết chặt chẽ đến sự hài lòng công việc, sự cam kết với tổ chức và ý định nghỉ việc, động lực làm việc, sáng kiến của nhân viên, hiệu quả làm việc của nhân viên, hiệu quả và kết quả hoạt động của tổ chức.

Người chủ động với những sáng kiến trong công việc được kỳ vọng sẽ có nhiều nỗ lực hơn trong quá trình tìm kiếm và đánh giá các cơ hội việc làm. Do đó, cùng với nhận thức về sự tương thích giá trị với tổ chức được chọn, những người chủ động cũng được kỳ vọng sẽ phát triển một nhận thức về sự tương thích giá trị với người quản lý của họ trong tổ chức đó. Do đó, các giả thuyết sau đây được đề xuất:

H1a: Tính cách chủ động có tác động tích cực đến nhận thức về sụ tương thích giá trị với tổ chức

\subsection{Mối quan hệ giữa tính cách chủ động và nhận thức sụ tương thích giá trị với người quản lý trục tiếp}

Sự chủ động gần như luôn gắn liền với bối cảnh xã hội và bị chi phối bởi phản ứng của người khác. Các đặc tính của lãnh đạo và các hành vi của họ rất quan trọng, dựa trên quan điểm điều hành, các nhà lãnh đạo hướng ngoại thường có các hành vi chi phối, quyết đoán và như thế họ đánh giá cao sự phục tùng và tuân thủ từ cấp dưới. Sự tương thích giá trị với người quản lý trực 
tiếp là sự giống nhau giữa hệ giá trị của lãnh đạo và nhân viên, điều này tạo ảnh hưởng tích cực đến thái độ và hành vi của nhân viên (Edwards \& Cable, 2009; Kristof-Brown et al., 2005). Trong một nghiên cứu định tính về hành vi cá nhân Cangiano, Parker, và Yeo (2019) nhận thấy rằng các phản ứng của người quản lý trực tiếp đóng vai trò quan trọng trong việc liệu những người có tính cách chủ động có phải là tài nguyên quan trọng của tổ chức. Vì vậy, nghiên cứu đề xuất giả thuyết:

H1b: Tính cách chủ động có liên quan tích cưc đến nhận thức về sụ tương thích giá trị với người quản lý

\subsection{Mối quan hệ giữa sụ tương thích giá trị với tổ chức và tương thích giá trị với người quản lý trục tiếp}

Lý thuyết tự quyết định (Deci \& Ryan, 1985) đề xuất rằng cá nhân sẽ có động lực mạnh mẽ khi hoạt động hoặc mục tiêu hành vi đã được tiếp thu trong phạm vi nó được chấp nhận là của riêng mình. Quá trình tham gia vào các giá trị, thái độ, hoặc cấu trúc điều tiết, như quy định bên ngoài của một hành vi được biến đổi thành một quy định hội nhập và do đó không còn đòi hỏi sự hiện diện của một nguồn lực bên ngoài. Theo đó, càng có nhiều giá trị tổ chức là tương thích với bản sắc hoặc giá trị của một người, thì càng có nhiều giá trị được thúc đẩy để đạt được giá trị được chia sẻ và mục tiêu dài hạn. Bởi vì người quản lý là đại diện của tổ chức (Eisenberger et al., 2010), các nhân viên có sự tương thích giá trị cao sẽ nỗ lực làm việc, đóng góp vào thành công chung của tổ chức và sẵn sàng chia sẻ với đồng nghiệp. Vì vậy, nghiên cứu đề xuất giả thuyết:

H1c: Sụ tương thích giá trị với tổ chức tác động tích cực đến sụ tuoong thích giá trị với người quản lý trục tiếp

\subsection{PP và $\mathrm{HB}$}

Tính cách chủ động là một trong những yếu tố dẫn đến sự thành công trong nghề nghiệp vì những người có tính cách chủ động có xu hướng tham gia vào hành vi tích cực (Seibert, Kraimer, \& Crant, 2001). Những cá nhân có khuynh hướng hành vi tích cực thường xuyên đề nghị tạo ra các cơ hội, cải thiện tình hình thông qua những ý tưởng, gợi ý sáng tạo về công việc, họ tham gia vào các hoạt động phát triển của công ty (có thể tham gia với mức độ nhiều hay ít) vì nhu cầu thành tích của họ (Thompson, 2005) và động lực học hỏi của họ (Major, Turner, \& Fletcher, 2006). Nghiên cứu của (Parker et al., 2010), và Li, Harris, Boswell, và Xie (2011) cho thấy rằng tính cách chủ động thể hiện hành vi chủ động ngay cả khi không có sự hỗ trợ của tình huống. Vì vậy nghiên cứu này kỳ vọng rằng khi tình huống không phù hợp với hành vi thì nhân viên có tính cách chủ động sẽ thể hiện hành vi chủ động.

Hành vi giúp đỡ là những nỗ lực cung cấp hỗ trợ và tạo điều kiện cho người khác (LePine \& Van Dyne, 1998), lên kế hoạch, hỗ trợ, tạo cơ hội giúp đỡ người khác thay vì chỉ đơn giản là đáp ứng các yêu cầu công việc. Hiện các nghiên cứu về hành vi giúp đỡ còn hạn chế và gần như mới chỉ ở mức giới thiệu định nghĩa và một nghiên cứu của (Waterwall, 2019) chỉ ra tác động tích cực của tính cách chủ động và hành vi chủ động (đảm nhiệm, hiệu quả nhiệm vụ, ngăn ngừa sai sót) chịu sự tác động trung gian của sự tập trung điều tiết (regulatory focus). Điều này cho thấy nghiên cứu về hành vi giúp đỡ còn đang bỏ ngõ, vì vậy nghiên cứu này lấp khoảng trống nghiên cứu bằng cách điều tra mối quan hệ giữa tính cách chủ động và hành vi giúp đỡ. Vì vậy, giả thuyết $\mathrm{H} 2$ được đề xuất như sau:

H2: Tính cách chủ động tác động đến hành vi giúp đõ

\subsection{Mối quan hệ giữa sụ tương thích giá trị với tổ chức và hành vi giúp đõ}

Các nghiên cứu đã tìm thấy mối quan hệ chặt chẽ giữa sự tương thích giá trị với tổ chức và hành vi công dân tổ chức như mức độ cam kết cao hơn, nghỉ việc giảm, sự hài lòng của nhân viên 
cao hơn, tăng hiệu quả tổ chức, sức khỏe cá nhân tốt hơn và tăng mức độ nhân viên thích cấp dưới, và người quản lý trực tiếp (Edwards \& Cable, 2009; Hoffman \& Woehr, 2006). Podsakoff, Whiting, Podsakoff, và Mishra (2011) đã cho thấy rằng các nhà quản lý có nhiều khả năng chọn các ứng viên có xu hướng thể hiện hành vi phù hợp với tổ chức. Việc biết liệu sự tương thích giá trị rất quan trọng trong việc dự đoán hành vi nhân viên ngay từ khâu tuyển chọn ứng viên gia nhập vào tổ chức. Vì vậy, giả thuyết sau đây được đề xuất. Vì vậy, các giả thuyết sau đây được đề xuất:

H3a: Sụ tương thích giá trị với tổ chức có tác động tích cực đến hành vi giúp đõ

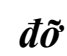

2.7. Mối quan hệ giữa sụ tương thích giá trị với nguời quản lý trục tiếp và hành vi giúp

Để thành công trong công việc, các nhân viên chủ động không chỉ có thể chọn tổ chức mà họ muốn làm việc, mà còn để phát triển một nhận thức (hoặc ít nhất là kỳ vọng) về sự tương thích giá trị với người quản lý của họ. Do đó, những nhân viên đó sẽ xem việc thể hiện hành vi chủ động như một cách hiệu quả để phát triển mối quan hệ tốt với người quản lý của mình để tạo điều kiện thuận lợi cho hiệu quả công việc cao. Giả định này cũng phù hợp với lý thuyết kỳ vọng (Vroom, 1964). Vì vậy, giả thuyết sau được đề xuất.

H3b: Sụ tương thích giá trị với người quản lý trục tiếp có tác động tích cực đến hành vi giúp đõ

\subsection{Vai trò trung gian của sụ tương thích giá trị với tổ chức và tương thích giá trị với người quản lý trục tiếp trong mối quan hệ giữa tính cách chủ động và hành vi giứp đõ}

Sự tương thích giá trị có vai trò quan trọng bởi nó tích hợp hai quan điểm lý thuyết đã tồn tại tương đối độc lập với nhau: lý thuyết về sự phù hợp cá nhân - môi trường và thiết kế công việc. Oldham và Hackman (2010) lưu ý rằng mô hình đặc điểm công việc ban đầu được xây dựng dựa trên giả định rằng các công việc có thể được mô tả như một tập hợp các nhiệm vụ tương đối độc lập với bối cảnh của chúng trong tổ chức. Bằng chứng cho thấy những người có định hướng vai trò linh hoạt thực sự có nhiều khả năng tham gia vào hành vi làm việc chủ động hơn (Parker, Williams, \& Turner, 2006).

Khi mục tiêu cá nhân của nhân viên phù hợp với mục tiêu của người quản lý thì nhân viên cảm thấy được kiểm soát công việc và được trao quyền cho những nỗ lực cá nhân trong các hoạt động có lợi cho tổ chức của họ (Vancouver \& Schmitt, 1991). Điều này sẽ giảm thiểu bất kỳ xung đột về chức năng đang tồn tại giữa lợi ích cá nhân và của tổ chức của họ (Colbert, Mount, Harter, Witt, \& Barrick, 2004; Eisenhardt, 1989). Vì vậy, nghiên cứu đề xuất giả thuyết:

H4: Sự tương thích giá trị với tổ chức và sự tương thích giá trị với người quản lý trục tiếp có vai trò trung gian trong mối quan hệ giữa tính cách chủ động và hành vi giúp đõ

Mặc dù, POV và PSV có vai trò trung gian riêng biệt trong mối quan hệ giữa $\mathrm{PP}$ và $\mathrm{HB}$, nhưng POV và PSV cũng có thể có mối quan hệ với nhau. Vì vậy nghiên cứu đề xuất giả thuyết:

H5: Mối quan hệ tích cực giũa tính cách chủ động và hành vi giúp đơ được điều tiết đa biến bởi POV và PSV

\section{Kết quả nghiên cứu}

\subsection{Mẫu nghiên cứu}

Ngày nay, điều dưỡng ở hầu hết các nước trên thế giới đều được công nhận là một nghề nghiệp độc lập, được bảo hộ bằng luật pháp, một số nước đã xây dựng luật hành nghề điều dưỡng. Tuy nhiên, tình hình dịch bệnh diễn biến phức tạp và sự quá tải của hệ thống bệnh viện ảnh hưởng 
xấu đến hiệu quả làm việc của các y tá. Ngoài ra, công việc của điều dưỡng viên có tính chất phụ thuộc lẫn nhau rất cao, họ thường trao đồi thông tin về nhiệm vụ của họ. Do đó, việc giúp đỡ lẫn nhau là rất cần thiết để đạt được tính phù hợp và hiệu quả trong công việc (Nickerson, 1992).

Với sự hỗ trợ của lãnh đạo và phòng nhân sự của các bệnh viện lớn ở Thành phố Hồ Chí Minh, nghiên cứu tiến hành thông qua khảo sát theo bảng câu hỏi đến bác sĩ và điều dưỡng viên bằng phương pháp chọn mẫu định lượng: phi xác suất - thuận tiện. Bảng khảo sát được gửi bảng khảo sát trực tiếp cho những người tình nguyện tham gia khảo sát. Bảng câu hỏi đặt ra các mục tiêu nghiên cứu, tự nguyện bản chất của khảo sát và đảm bảo tính bảo mật những người tham gia và được sự đồng ý của những người tham gia khảo sát. Dữ liệu của chúng tôi được thu thập từ Tháng 01 đến tháng 04 năm 2020, với 715 mẫu thu thập từ 800 bảng khảo sát phát ra $(98,38 \%)$. Chi tiết thống kê mô tả mẫu khảo sát được trình bày trong Bảng 1 .

\section{Bảng 1}

Thống kê mô tả mẫu khảo sát

\begin{tabular}{|c|c|c|c|c|c|c|c|}
\hline \multicolumn{2}{|c|}{ Mẫu n = 715} & Tần số & $\%$ & \multicolumn{2}{|c|}{ Mẫu n = 715} & Tần số & $\%$ \\
\hline \multirow{4}{*}{ Tuổi } & $<25$ & 257 & 35,94 & \multirow{3}{*}{ Thâm niên } & $<5$ năm & 244 & 34,13 \\
\hline & $25-35$ & 270 & 37,76 & & 5- 10 năm & 359 & 50,21 \\
\hline & $36-45$ & 129 & 18,04 & & > 5 năm & 112 & 15,66 \\
\hline & $>45$ & 59 & 8,25 & \multirow{4}{*}{$\begin{array}{c}\text { Trình độ } \\
\text { học vấn }\end{array}$} & Trung cấp & 124 & 17,3 \\
\hline \multirow{5}{*}{$\begin{array}{l}\text { Thu } \\
\text { nhập }\end{array}$} & < 5 triệu & 119 & 16,64 & & Cao đẳng & 441 & 61,7 \\
\hline & $5-<10$ triệu & 336 & 46,99 & & Đại học & 102 & 14,3 \\
\hline & $10-<15$ triệu & 154 & 21,54 & & Sau đại học & 48 & 6,7 \\
\hline & \multirow{2}{*}{ > 15triệu } & \multirow{2}{*}{106} & \multirow{2}{*}{14,83} & \multirow{2}{*}{ Giới tính } & Nữ & 513 & 71,75 \\
\hline & & & & & Nam & 202 & 28,25 \\
\hline
\end{tabular}

Nguồn: Kết quả phân tích dữ liệu của tác giả (2020)

\subsection{Thang do}

Thang đo trong nghiên cứu được xây dựng dựa trên cơ sở lý thuyết về tính cách chủ động (Bateman \& Crant, 1993), giá trị tương thích với tổ chức và giá trị tương thích với người quản lý trực tiếp (Nguyen, 2013), và chủ động giúp đỡ (Parker \& Collins, 2010). Để đảm bảo hiệu quả khi chuyển thể sang tiếng Việt, nghiên cứu đã tiến hành dịch thuật và dịch ngược lại song song (doubleblind way). Sau đó, chúng tôi đã thảo luận và hiệu chỉnh bản dịch với các chuyên gia nhân sự và nhân viên y tế để lại cho phù hợp với tình hình tại Việt Nam. Các biến quan sát được khảo sát theo thang đo Likert 5 mức độ ranging from 1 (rất không đồng ý) to 5 (rất đồng ý).

Tính cách chủ động (ký hiệu PP): Thang đo này được đo lường bằng 10 biến quan sát của (Bateman \& Crant, 1993). Độ tin cậy của thang đo là 0,947.

Sự tương thích giá trị với tổ chức (ký hiệu POV): Thang đo này sử dụng thang đo của (Nguyen, 2013) được đo lường bằng 06 biến quan sát. Độ tin cậy của thang đo là 0,883 .

Sự tương thích giá trị với người quản lý trực tiếp (ký hiệu PSV): Thang đo khái niệm giá trị tương thích với người quản lý trực tiếp sử dụng thang đo của Nguyen (2013) được đo lường bằng 06 biến quan sát. Độ tin cậy của thang đo là 0,863 . 
Hành vi giúp đỡ (ký hiệu HB): Thang đo hành vi giúp đỡ được đo lường bằng 07 biến quan sát (LePine \& Van Dyne, 1998). Độ tin cậy của thang đo là $0,810$.

\section{Kết quả}

\subsection{Thống kê mô tả và tưong quan giữa các biến}

Bảng 2 thể hiện giá trị trung bình, độ lệch chuẩn của các biến trong mô hình. Tất cả các chỉ số tương quan (correlations) đều nhỏ hơn 0,5 cho thấy không có hiện tượng đa cộng tuyến (multicollinearity). Kiểm định Pearson ở bảng 2 cho thấy hầu hết các biến độc lập tương quan với biến phụ thuộc ở mức ý nghĩa $99 \%$, các hệ số tương quan đều $>0,3$. Độ giá trị phân biệt: thông qua nhân tố khám phá với các biến cấu trúc trong các nhân tố, kết quả phân tích định lượng cho thấy hệ số tải của các nhân tố này lớn hơn hệ số tải của các nhân tố khác.

\section{Bảng 2}

Độ tin cậy của thang đo, giá trị trung bình $(\mathrm{M})$, độ lệch chuẩn $(\mathrm{SD})$ và tương quan giữa các biến

\begin{tabular}{lccccccc}
\hline \multicolumn{1}{c}{ Biến } & Cronbach's $\boldsymbol{\alpha}$ & M & SD & $\mathbf{1}$ & $\mathbf{2}$ & $\mathbf{3}$ & $\mathbf{4}$ \\
\hline 1. PP &, 913 & 3,522 &, 63 & 1 & & & \\
2. POV &, 876 & 3,631 &, 70 &, $472 * *$ & 1 & & \\
3. PSV &, 859 & 3,444 &, 73 &, $439 * *$ &, $493 * *$ & 1 & \\
4. HB &, 923 & 3,618 &, 73 &, $490 * *$ &, $459 * *$ &, $491 * *$ & 1 \\
\hline
\end{tabular}

Ghi chú: * Độ tin cậy $p<0,001, * *$ độ tin cậy $p<0,005$

Nguồn: Kết quả phân tích dữ liệu của tác giả (2020)

\subsection{Phân tích nhân tố khẳng định (Confirmatory Factor Analyses - CFA)}

Khi thực hiện phân tích nhân tố khám phá với các thang đo trong mô hình nghiên cứu đề xuất, nghiên cứu sử dụng phương pháp rút trích Principal Axis Factoring cùng với phương pháp quay Promax. Kết quả phân tích nhân tố khám phá thu được như sau: Hệ số KMO $=0,945$ thoả mãn điều kiện nằm trong đoạn $[0,5 ; 1,0]$, hệ số Sig. của kiểm định Bartlett's là 0,000 nhỏ hơn $1 / 1000$ cho thấy các biến này có độ kết dính với nhau và hoàn toàn phù hợp với phân tích nhân tố. Với 4 nhân tố có được, nghiên cứu sinh sẽ thực hiện phân tích CFA. Với các chỉ số: Chi-square/df $=2,331(<3), \mathrm{RMSEA}=0,043(<, 08), \mathrm{GFI}=0,918, \mathrm{TLI}=0,952, \mathrm{CFI}=0,956>0,9)$ điều đạt tiêu chuẩn. Kết quả này cho thấy các chỉ số mô hình đo lường phù hợp với dữ liệu, như vậy mô hình xác nhận 4 nhân tố. Về mặt hội tụ, chúng tôi đã tính toán độ tin cậy tổng hợp (composite reliability - CR) và phương sai trích (Average Variance Extracted - AVE). Kết quả cho thấy CR của mỗi nhân tố nằm trong khoảng từ 0,858 đến 0,946 lớn hơn tiêu chuẩn 0,70 . AVE cho mỗi nhân tố dao động từ 0,502 đến 0,546 lớn hơn 0,5 . Vì vậy, các biến trong mô hình đạt giá trị phân biệt và hội tụ được xác nhận. 


\section{Bảng 3}

Các chỉ số CFA, CR, AVE

\begin{tabular}{|c|c|c|c|c|c|c|c|}
\hline Chỉ số & $\begin{array}{c}\text { Ngưỡng } \\
\text { chấp } \\
\text { nhận }\end{array}$ & $\begin{array}{l}\text { Số liệu } \\
\text { AMOS }\end{array}$ & Đánh giá & Chỉ số & $\begin{array}{c}\text { Ngưỡng } \\
\text { chấp } \\
\text { nhận }\end{array}$ & $\begin{array}{l}\text { Số liệu } \\
\text { AMOS }\end{array}$ & Đánh giá \\
\hline \multicolumn{4}{|c|}{ Độ tin cậy tổng họ̣p (CR) } & \multicolumn{4}{|c|}{ Phương sai trích (AVE) } \\
\hline PP & \multirow{4}{*}{$>0,7$} & 0,946 & Chấp nhận & PP & \multirow{4}{*}{$>0,5$} & 0,523 & $\begin{array}{l}\text { Chấp } \\
\text { nhận }\end{array}$ \\
\hline POV & & 0,879 & Chấp nhận & POV & & 0,509 & $\begin{array}{l}\text { Chấp } \\
\text { nhận }\end{array}$ \\
\hline PSV & & 0,858 & Chấp nhận & PSV & & 0,502 & $\begin{array}{l}\text { Chấp } \\
\text { nhận }\end{array}$ \\
\hline $\mathrm{HB}$ & & 0,878 & Chấp nhận & $\mathrm{HB}$ & & 0,546 & $\begin{array}{l}\text { Chấp } \\
\text { nhận }\end{array}$ \\
\hline \multicolumn{8}{|c|}{ Chỉ số CFA } \\
\hline$\chi^{2 / \mathrm{df}}$ & GFI & \multicolumn{2}{|c|}{ CFI } & \multicolumn{2}{|c|}{ TLI } & \multicolumn{2}{|c|}{ RMSEA } \\
\hline 2,331 & 0,918 & \multicolumn{2}{|c|}{,956 } & \multicolumn{2}{|l|}{0,952} & \multicolumn{2}{|c|}{,043 } \\
\hline
\end{tabular}

Nguồn: Kết quả phân tích dữ liệu của tác giả (2020)

\subsection{Kiểm định giả thuyết nghiên cúu}

Bảng 4 tóm tắt kết quả hồi quy bội. Nghiên cứu sử dụng qui trình phân tích biến trung gian được đề xuất bởi (Baron \& Kenny, 1986). Kết quả cho thấy PP, POV, PSV và HB có mối liên hệ mật thiết với nhau. Các giả thuyết được đề xuất đã được phân tích hồi quy bội và phân tích trung gian với sự trợ giúp của phương pháp phân tích bootstrap (Hayes \& Preacher, 2013). Tất cả các mô hình hồi qui đạt chuẩn theo thống kê $\mathrm{F}$.

Mô hình 1 và mô hình 2 cho thấy tác động trực tiếp của $P P$ đến $\operatorname{POV}(\beta=0,463, p=0,000)$ và $\operatorname{PSV}(\beta=0,418, p=0,000)$. Do đó, những kết quả này hỗ trợ cho giả thuyết $\mathrm{H} 1 \mathrm{a}, \mathrm{H} 1 \mathrm{~b}$. Mô hình 3 cho thấy tác động trực tiếp của POV đến PSV $(\beta=0,406, p=0,000)$, nên giả thuyết H1c được chấp nhận. Mô hình 4 cho thấy tác động trực tiếp của $P P$ đến $H B(\beta=0,458, p=0,000)$. Vì vậy giả thuyết $\mathrm{H} 2$ được chấp nhận. Mô hình 5 và mô hình 6 thể hiện tác động trực tiếp của các biến trung gian là $\operatorname{POV}(\beta=0,425, p=0,000)$, và $\operatorname{PSV}(\beta=0,343, p=0,000)$ đến $\mathrm{HB}$. Kết quả này cho thấy giả thuyết $\mathrm{H} 3 \mathrm{a}$ và $\mathrm{H} 3 \mathrm{~b}$ được chấp nhận. Ngoài ra, nghiên cứu sử dụng bootstrap để kiểm tra tác động trực tiếp và gián tiếp của $P P$ đến $H B$.

Kết quả bootstrap được thể hiện trong bảng 5 và 6 . Nghiên cứu sử dụng Process macro (mô hình 6) để kiểm định tác động của PP thông qua POV, PSV đến HB. Mô hình 10 đại diện cho tổng tác động của $\mathrm{PP}$ đến $\mathrm{HB}(\beta=0,363, \mathrm{p}=0,000)$. Do đó giả thuyết $\mathrm{H} 2$ được chấp nhận. Tất cả các số liệu thống kê F đều có ý nghĩa.

Dựa trên kết quả bootstrap với 2.000 mẫu lặp lại với khoảng tin cậy $95 \%$ không có số 0 giữa các tác động gián tiếp. Tất cả các tác động gián tiếp cho thấy POV, PSV có vai trò trung gian trong mối quan hệ giữa $\mathrm{PP}$ và $\mathrm{HB}$ (Bảng 6). Do đó, giả thuyết $\mathrm{H} 4$ được chấp nhận. Tương tự, không có số 0 giữa các tác động đa biến trung gian của POV và PSV trong mối quan hệ giữa $P P$ và $\mathrm{HB}$. Do đó, giả thuyết H5 được chấp nhận. 


\section{Bảng 4}

Kết quả tác động của các nhân tố trong mô hình nghiên cứu

\begin{tabular}{|c|c|c|c|c|c|c|}
\hline \multirow{2}{*}{ Biến } & \multirow{2}{*}{$\begin{array}{c}\text { POV } \\
\text { Mô hình } 1\end{array}$} & \multicolumn{2}{|c|}{ PSV } & \multicolumn{3}{|c|}{ HB } \\
\hline & & Mô hình 2 & Mô hình 3 & Mô hình 4 & Mô hình 5 & Mô hình 6 \\
\hline PP &, $463 * *$ &, $418 * *$ & - & $458 * *$ & - & - \\
\hline POV & - & - &, $406 * *$ & - &, $425 * *$ & - \\
\hline PSV & - & - & - & - & - &, $343 * *$ \\
\hline $\mathrm{R}^{2}$ & ,440 & ,382 &, 367 & ,433 & ,390 &, 117 \\
\hline $\mathrm{R}^{2}$ hiệu chỉnh & ,439 & ,381 &, 366 & ,432 & ,390 &, 116 \\
\hline $\mathrm{F}$ & $560,63 * *$ & $441,00 * *$ & $413,21 * *$ & $544,67 * *$ & $456,56 * *$ & $94,82 * *$ \\
\hline
\end{tabular}

Ghi chú: ${ }^{* *} \mathrm{p} \leq 0.001 ; * \mathrm{p} \leq 0.05$

Nguồn: Kết quả phân tích dữ liệu của tác giả (2020)

\section{Bảng 5}

Kết quả phân tích mô hình trung gian đa biến bằng PROCESS macro

\begin{tabular}{|l|r|r|r|r|}
\hline \multirow{2}{*}{} & \multicolumn{1}{|c|}{ POV } & \multicolumn{1}{c|}{ PSV } & \multicolumn{2}{c|}{ HB } \\
\cline { 2 - 5 } & \multicolumn{1}{|c|}{ Mô hình 7 } & \multicolumn{1}{c|}{ Mô hình 8 } & Mô hình 9 & \multicolumn{1}{c|}{ Mô hình 10 } \\
\hline PP &, $423 * *(, 031)$ &, $397 * *(, 038)$ &, $323^{* *}(, 038)$ &, $363 * *(, 383)$ \\
\hline POV & - &, $321^{* *}(, 035)$ &, $278^{* *}(, 035)$ & - \\
\hline PSV & - & - &, $164 * *(, 035)$ &, 675 \\
\hline R &, 668 &, 623 &, 722 &, 434 \\
\hline $\mathrm{R}^{2}$ &, 446 &, 498 &, 421 & $92,57 * *$ \\
\hline F & $95,11 * *$ & $92,45 * *$ & $95,93 * *$ & - \\
\hline
\end{tabular}

Ghi chú: Số trong dấu () đại diện cho sai số chuẩn (standard error); Mô hình 10 thể hiện tổng tác động của PP đến HB và mô hình 9 thể hiện tác động của các biến trung gian trong mối quan hệ giữa $\mathrm{PP}$ và $\mathrm{HB}$; **p \# 0,001 ; và *p \# 0,05 Nguồn: Kết quả phân tích dữ liệu của tác giả (2020)

\section{Bảng 6}

Tác động của PP đến HB thông qua các biến trung gian

\begin{tabular}{|l|l|r|r|r|r|}
\hline & \multicolumn{1}{|c|}{ Tác động gián tiếp } & \multicolumn{1}{|c|}{$\begin{array}{c}\text { Mức tác động } \\
\text { gián tiếp }\end{array}$} & SE & Boot LLCI & Boot ULCI \\
\hline Tổng & &, 306 &, 040 &, 225 &, 385 \\
\hline Ind1 & Tác động của: PP $\rightarrow$ POV $\rightarrow$ PSV &, 203 &, 039 &, 132 &, 279 \\
\hline Ind2 & PP $\rightarrow$ POV $\rightarrow$ PSV $\rightarrow$ HB &, 065 &, 021 &, 027 &, 107 \\
\hline Ind3 & PP $\rightarrow$ PSV $\rightarrow$ HB &, 038 &, 013 &, 015 &, 065 \\
\hline
\end{tabular}

Nguồn: Kết quả phân tích dữ liệu của tác giả (2020)

Ghi chú: Ind1-Ind3 thể hiện tác động gián tiếp từ kết quả phân tích bootstrap. Boot LLCI thể hiện khoản tin cậy cận dưới và boot ULCI thể hiện khoản tin cậy cận trên. 
Hình 1 thể hiện tác động của các yếu tố trong mô hình.

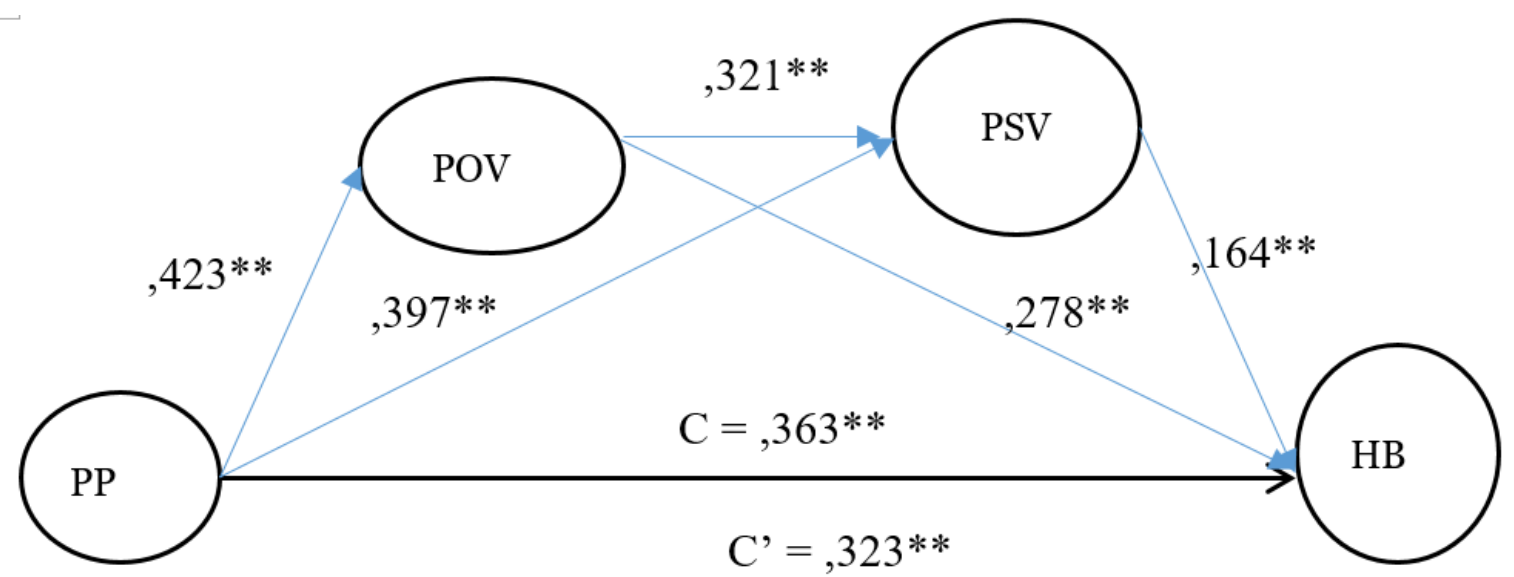

Hình 1. Mô hình nghiên cứu

Nguồn: Kết quả phân tích dữ liệu của tác giả (2020)

\section{Thảo luận kết quả}

Trong môi trường cạnh tranh ngày nay, hành vi giúp đỡ rất quan trọng trong tổ chức. Các tổ chức đang chú trọng thúc đẩy hành vi này vì những ưu điểm vượt trội của nó. Nghiên cứu đã tìm hiểu mối quan hệ giữa $\mathrm{PP}$ và $\mathrm{HB}$ được trung gian bởi $\mathrm{POV}$ và $\mathrm{PSV}$. Phát hiện quan trọng đầu tiên của nghiên cứu là $P P$ có tác động tích cực đến $H B$. Nhân viên có tính cách chủ động có xu hướng thể hiện các hành vi giúp đỡ. Kết quả này tương đồng với các nghiên cứu trước (Fuller \& Marler, 2009; Fuller, Marler, \& Hester, 2012; Parker \& Collins, 2010).

Kết quả kiểm định vai trò đa trung gian của POV, PSV trong mối quan hệ giữa $\mathrm{PP}$ và $\mathrm{HB}$. Những kết quả của nghiên cứu có thể được giải thích theo lập luận của thuyết tự xác định (Selfdetermination theory) và sự phù hợp giữa cá nhân với tổ chức (Person- Organization Fit - P-O F) rằng nhận thức của con người về môi trường có thể tác động đến hành vi. Kết quả mô hình đa trung gian cho thấy POV, PSV có vai trò trung gian tác động tích cực đến mối quan hệ giữa $P P$ và HB. Mô hình đa biến trung gian này cho thấy sự tương thích giá trị với tổ chức được tác động bởi tính cách chủ động có tác động tích cực trực tiếp đến hành vi giúp đỡ và tác động tích cực gián tiếp đến hành vi giúp đỡ thông qua sự tương thích giá trị với người quản lý trực tiếp. Hiện nay mô hình đa biến trung gian về mối quan hệ giữa $\mathrm{PP}, \mathrm{POV}, \mathrm{PSV}, \mathrm{HB}$ vẫn chưa được nghiên cứu nhiều nên đây là điểm đóng góp chính của nghiên cứu trong cơ sở lý thuyết về khoa học hành vi.

\section{Kết luận}

\subsection{Kết luận và hàm ý quản trị}

PP đã được tìm thấy có tác động đáng kể đến kết quả hành vi và tổ chức của nhân viên. Do đó, nghiên cứu này bổ sung vào nghiên cứu trước đây khi nó khám phá ra ngoài những ảnh hưởng của điều kiện nơi làm việc để giải thích cách $P P$ dẫn đến hành vi chủ động. Đầu tiên, nghiên cứu của chúng tôi đã kết hợp $\mathrm{PP}$ vào khung nghiên cứu lần đầu tiên, xác nhận rằng PP là một yếu tố thúc đẩy hành vi giúp đỡ. Thứ hai, bằng cách kiểm tra các tác động của $\mathrm{POV}, \mathrm{PSV}$ phù hợp với vai trò là trung gian của mối quan hệ giữa $\mathrm{PP}$ và $\mathrm{HB}$, nghiên cứu hiện tại đóng góp vào cơ sở lý thuyết về sự tương thích giá trị. Cụ thể, chúng tôi đóng góp cho lý thuyết về sự tương thích giá trị bằng cách chứng minh sự kết nối của $\mathrm{PP}$ với không chỉ tác động đến sự tương thích giá trị với tổ chức, mà còn tương thích với người quản lý. Hầu hết các nghiên cứu thực nghiệm trước đây đã xem xét ảnh hưởng của sự tương thích giá trị với tổ chức (Seong \& Choi, 2014); (Pierro, Sheveland, Livi, \& Kruglanski, 2015), rất ít nghiên cứu đã khám phá sự tương thích giá trị với cả tổ chức và người 
quản lý trực tiếp. Do đó, chúng tôi đã tích hợp chúng thành một mô hình mạch lạc, trong đó sự tương thích giá trị với tổ chức và người quản lý trực tiếp đóng vai trò là một cơ chế định hướng nhiệm vụ và sự hỗ trợ của tổ như một cơ chế định hướng quan hệ này. Cuối cùng, kết quả cho thấy những nhân viên có tính cách chủ động sẽ thể hiện sự tương thích, phù hợp với tổ chức và người quản lý cao hơn so với những người khác. Việc điều chỉnh này có thể được thực hiện thông qua việc giúp đỡ đồng nghiệp nhìn thấy được những vấn đề và các cách thức hỗ trợ làm việc chung. Điều này cũng thể hiện được sự phù hợp với lý thuyết và các nghiên cứu thực nghiệm khi nhân viên họ cảm thấy trách nhiệm mang lại thay đổi tích cực (Fuller, Marler, \& Hester, 2006).

Trong mối quan hệ tính cách chủ động tác động đến giá trị tương thích của tổ chức và người quản lý trực tiếp và cùng chiều cho thấy những nhân viên có tính cách chủ động càng cao thì càng có sự tương thích với tổ chức và người quản lý càng nhiều. Để phát huy được điều này thì các nhà quản lý ở bệnh viện cần tìm kiếm và chọn lựa những nhân viên điều dưỡng có tính chủ động ngay từ ban đầu. Ngoài ra, người quản lý trực tiếp nên được phép tham gia vào quá trình phỏng vấn, vì điều này sẽ giúp bệnh viện tìm ra nhân viên điều dưỡng phù hợp với mình cũng như có thể nắm bắt được suy nghĩ, tính cách, nguyện vọng của ứng viên. Đồng thời, ứng viên cũng có thể có cách nhìn ban đầu về người quản lý trực tiếp của mình như thế nào.

Về mối quan hệ giữa tính cách chủ động tác động tích cực đến hành vi giúp đỡ đồng nghiệp của các điều dưỡng viên có thể ứng dụng trong môi trường làm việc lúc nào cũng có sự cạnh tranh nhưng bệnh viện cần phải xây dựng cạnh tranh công bằng tại nơi làm việc như khuyến khích hỗ trợ, giúp đỡ giải quyết công việc chung nhưng cạnh tranh công bằng trong hoàn thành các chỉ tiêu đề ra.

\subsection{Hạn chế và hướng nghiên cứu tiếp theo}

Ngoài những đóng góp về lý thuyết và thực tiễn như đã nêu, nghiên cứu này vẫn còn một số hạn chế như sau. Thứ nhất, vì dữ liệu trong nghiên cứu chỉ thu thập từ một nguồn là các nhân viên điều dưỡng tại các bệnh viên ở Thành phố Hồ Chí Minh, điều này có thể gây ra phương sai chung phương pháp tiềm năng. Để củng cố tính tổng quát của các phát hiện của chúng tôi, nghiên cứu nhân rộng nên được thực hiện trong các bối cảnh khác nhau. Bên cạnh đó, với kích thước mẫu lớn của nghiên cứu, việc phát hiện các mối quan hệ quan trọng là tương đối dễ dàng hơn, vì vậy các phát hiện thực nghiệm cần nhiều nghiên cứu chuyên sâu hơn để cung cấp thêm ý nghĩa lý thuyết.

Thứ hai, trong tương lai, hành vi chủ động nên được các đáp viên khác đánh giá như lãnh đạo và đồng nghiệp đánh giá.

Cuối cùng, kết quả của nghiên cứu chỉ giới hạn ở một hành vi giúp đỡ. Nghiên cứu trong tương lai có thể kiểm tra một phạm vi rộng hơn của hành vi công việc, đặc biệt là các kết quả liên quan đến hiệu suất, chẳng hạn như hiệu suất trong vai trò và ngoài vai trò công việc.

\section{Tài liệu tham khảo}

Ajzen, I. (1991). The theory of planned behavior. Organizational Behavior and Human Decision Processes, 50(2), 179-211.

Allen, M., Dietz, M., Blair, K. S., van Beek, M., Rees, G., Vestergaard-Poulsen, P., \& Roepstorff, A. (2012). Cognitive-affective neural plasticity following active-controlled mindfulness intervention. The Journal of Neuroscience: The Official Journal of the Society for Neuroscience, 32(44), 15601-15610.

Bakker, A. B., \& Demerouti, E. (2007). The job demands-resources model: State of the art. Journal of Managerial Psychology, 22(3), 309-328. 
Bardi, A., \& Schwartz, S. H. (2003). Values and behavior: Strength and structure of relations. Personality \& Social Psychology Bulletin, 29(10), 1207-1220.

Baron, R. M., \& Kenny, D. A. (1986). The moderator-mediator variable distinction in social psychological research: Conceptual, strategic, and statistical considerations. Journal of Personality and Social Psychology, 51(6), 1173-1182.

Bateman, T. S., \& Crant, J. M. (1993). The proactive component of organizational behavior: A measure and correlates. Journal of Organizational Behavior, 14(2), 103-118.

Bindl, U. K., \& Parker, S. K. (2011). Proactive work behavior: Forward-thinking and changeoriented action in organizations. In APA handbooks in psychology®. APA handbook of industrial and organizational psychology, Vol 2: Selecting and developing members for the organization (pp. 567-598). Washington, DC: American Psychological Association.

Cangiano, F., Parker, S. K., \& Yeo, G. B. (2019). Does daily proactivity affect well-being? The moderating role of punitive supervision. Journal of Organizational Behavior, 40(1), 59-72.

Chen, C.-Y., Yen, C.-H., \& Tsai, F. C. (2014). Job crafting and job engagement: The mediating role of person-job fit. International Journal of Hospitality Management, 37(1), 21-28.

Christian, M. S., Garza, A. S., \& Slaughter, J. E. (2011). Work engagement: A quantitative review and test of its relations with task and contextual performance. Personnel Psychology, 64(1), 89-136.

Colbert, A. E., Mount, M. K., Harter, J. K., Witt, L. A., \& Barrick, M. R. (2004). Interactive effects of personality and perceptions of the work situation on workplace deviance. The Journal of Applied Psychology, 89(4), 599-609.

Deci, E. L., \& Ryan, R. M. (1985). Intrinsic motivation and self-determination in human behavior. Boston, MA: Springer US.

DuBrin, A. J. (2012). Leadership: Research findings, practice, and skills. Boston, MA: Cengage Learning.

Edwards, J. R., \& Cable, D. M. (2009). The value of value congruence. Journal of Applied Psychology, 94(3), 654-677.

Eisenberger, R., Karagonlar, G., Stinglhamber, F., Neves, P., Becker, T. E., Gonzalez-Morales, M. G., \& Steiger-Mueller, M. (2010). Leader-member exchange and affective organizational commitment: The contribution of supervisor's organizational embodiment. The Journal of Applied Psychology, 95(6), 1085-1103.

Eisenhardt, K. M. (1989). Building theories from case study research. The Academy of Management Review, 14(4), 532-550.

Fuller, B., Jr., \& Marler, L. E. (2009). Change driven by nature: A meta-analytic review of the proactive personality literature. Journal of Vocational Behavior, 75(3), 329-345.

Fuller, J. B., Marler, L. E., \& Hester, K. (2006). Promoting felt responsibility for constructive change and proactive behavior: Exploring aspects of an elaborated model of work design. Journal of Organizational Behavior, 27(8), 1089-1120.

Fuller, J. B., Marler, L. E., \& Hester, K. (2012). Bridge building within the province of proactivity. Journal of Organizational Behavior, 33(8), 1053-1070.

Grant, A. M., \& Ashford, S. J. (2008). The dynamics of proactivity at work. Research in Organizational Behavior, 28(1), 3-34. 
Griffin, M. A., Neal, A., \& Parker, S. K. (2007). A new model of work role performance: Positive behavior in uncertain and interdependent contexts. Academy of Management Journal, 50(2), 327-347.

Hayes, A. F., \& Preacher, K. J. (2013). Conditional process modeling: Using structural equation modeling to examine contingent causal processes. In Quantitative methods in education and the behavioral sciences: Issues, research, and teaching. Structural equation modeling: A second course (2nd ed.) (pp. 219-266). Charlotte, NC: IAP Information Age Publishing.

Hoffman, B. J., \& Woehr, D. J. (2006). A quantitative review of the relationship between personorganization fit and behavioral outcomes. Journal of Vocational Behavior, 68(3), 389-399.

Kristof-Brown, A. L., Zimmerman, R. D., \& Johnson, E. C. (2005). Consequences of individual's fit at work: A meta-analysis of person-job, person-organization, person-group, and personsupervisor fit. Personnel Psychology, 58(2), 281-342.

LePine, J. A., \& Van Dyne, L. (1998). Predicting voice behavior in work groups. Journal of Applied Psychology, 83(6), 853-868.

Lewin, K., \& Cartwright, D. (1964). Field theory in social science: Selected theoretical papers (1st ed.). New York, NY: Harper \& Row.

Li, N., Harris, T. B., Boswell, W. R., \& Xie, Z. (2011). The role of organizational insiders' developmental feedback and proactive personality on newcomers' performance: An interactionist perspective. The Journal of Applied Psychology, 96(6), 1317-1327.

Major, D. A., Turner, J. E., \& Fletcher, T. D. (2006). Linking proactive personality and the Big Five to motivation to learn and development activity. The Journal of Applied Psychology, 91(4), 927-935.

McCormick, B. W., Guay, R. P., Colbert, A. E., \& Stewart, G. L. (2019). Proactive personality and proactive behaviour: Perspectives on person-situation interactions. Journal of Occupational and Organizational Psychology, 92(1), 30-51.

Ng, T. W. H., \& Feldman, D. C. (2012). Employee voice behavior: A meta-analytic test of the conservation of resources framework. Journal of Organizational Behavior, 33(2), 216-234.

Ng, T. W. H., \& Feldman, D. C. (2014). Subjective career success: A meta-analytic review. Journal of Vocational Behavior, 85(2), 169-179.

Nguyen, K. T. (2013). The relationship between proactive personality and performance: Why and when? Retrieved January 01, 2020, from https://search.proquest.com/docview/ 1444656381/abstract/E4CC25F18D98472APQ/1

Nickerson, R. S. (1992). Looking ahead: Human factors challenges in a changing world (1st ed.). Hillsdale, NJ: CRC Press.

Oldham, G. R., \& Hackman, J. R. (2010). Not what it was and not what it will be: The future of job design research. Journal of Organizational Behavior, 31(2/3), 463-479.

Parker, S. K., Bindl, U. K., \& Strauss, K. (2010). Making things happen: A model of proactive motivation. Journal of Management, 36(4), 827-856.

Parker, S. K., \& Collins, C. G. (2010). Taking stock: Integrating and differentiating multiple proactive behaviors. Journal of Management, 36(3), 633-662.

Parker, S. K., Williams, H. M., \& Turner, N. (2006). Modeling the antecedents of proactive behavior at work. Journal of Applied Psychology, 91(3), 636-652. 
Pierro, A., Sheveland, A., Livi, S., \& Kruglanski, A. W. (2015). Person-group fit on the need for cognitive closure as a predictor of job performance, and the mediating role of group identification. Group Dynamics: Theory, Research, and Practice, 19(2), 77-90.

Podsakoff, N. P., Whiting, S. W., Podsakoff, P. M., \& Mishra, P. (2011). Effects of organizational citizenship behaviors on selection decisions in employment interviews. The Journal of Applied Psychology, 96(2), 310-326.

Rauch, A., \& Frese, M. (2007). Let's put the person back into entrepreneurship research: A metaanalysis on the relationship between business owners' personality traits, business creation, and success. European Journal of Work and Organizational Psychology, 16(4), 353-385.

Schwartz, S. H. (1992). Universals in the content and structure of values: Theoretical advances and empirical tests in 20 countries. Advances in Experimental Social Psychology, 25(1), 1-65.

Schwartz, S. H. (1996). Value priorities and behavior: Applying a theory of integrated value systems. In The ontario symposium on personality and social psychology, Vol. 8. The psychology of values: The ontario symposium (pp. 1-24). Hillsdale, NJ: Lawrence Erlbaum Associates, Inc.

Schwartz, S. H., Cieciuch, J., Vecchione, M., Davidov, E., Fischer, R., Beierlein, C., \& Konty, M. (2012). Refining the theory of basic individual values. Journal of Personality and Social Psychology, 103(4), 663-688.

Seibert, S. E., Kraimer, M. L., \& Crant, J. M. (2001). What do proactive people do? A longitudinal model linking proactive personality and career success. Personnel Psychology, 54(4), 845-874.

Seong, J. Y., \& Choi, J. N. (2014). Effects of group-level fit on group conflict and performance: The initiating role of leader positive affect. Group \& Organization Management, 39(2), 190-212.

Tett, R. P., \& Burnett, D. D. (2003). A personality trait-based interactionist model of job performance. Journal of Applied Psychology, 88(3), 500-517.

Thompson, J. A. (2005). Proactive personality and job performance: A social capital perspective. Journal of Applied Psychology, 90(5), 1011-1017.

Vancouver, J. B., \& Schmitt, N. W. (1991). An exploratory examination of person-organization fit: Organizational goal congruence. Personnel Psychology, 44(2), 333-352.

Vroom, V. H. (1964). Work and motivation. Oxford, UK: Wiley.

Vveinhardt, J., \& Gulbovaite, E. (2013). Comparative analysis of the researches on personal and organizational value congruence. Management of Organizations Systematic Research, 67, 127-142. doi:10.7720/MOSR.1392-1142.2013.67.9

Vveinhardt, J., \& Gulbovaite, E. (2014). Diagnostic instruments for value congruence. Journal of Business Theory and Practice, 2(2), 126-151.

Waterwall, B. (2019). Linking proactive personality and proactive behavior: The mediating effect of regulatory focus. Journal of Organizational Psychology, 19(1), 108-122.

Xu, M., Qin, X., Dust, S. B., \& DiRenzo, M. S. (2019). Supervisor-subordinate proactive personality congruence and psychological safety: A signaling theory approach to employee voice behavior. The Leadership Quarterly, 30(4), 440-453. 